\title{
Study of hormone levels and semen parameters in infertile patients and fertile controls from Batna
}

\author{
Type of article: Conference abstract \\ Bousnane Nour El Houda*, Chennaf Ali, Yahia Mouloud \\ University Batna, Faculty of natural and life sciences, Laboratory of Biotechnology of \\ Bioactive Molecules and Cellular Pathophysiology \\ hodanorel20@hotmail.fr
}

\begin{abstract}
Background: Male infertility or the inability of a man to procreate is a major public health problem and is a leading cause of marital discord in countries such as Algeria. Endocrine disorders that can be associated with significant medical pathology remain an important factor to be considered in the etiology of male infertility, and those disorders are usually associated with alteration of sperm count or in severe cases with azoospermia. In this study, we are evaluating semen parameters and hormonal levels of patients with idiopathic male infertility and comparing their results with healthy fertile controls.
\end{abstract}

Methods: We aim to study the impact of hormones; follicle stimulating hormone (FSH), luteinizing hormone (LH), testosterone (T) and prolactin (PRL) on idiopathic male fertility and their impact on semen parameters, we conducted a study on 71 male subjects with 39 infertile men and 32 fertile controls from the city of Batna (Algeria). Our study lasted six months and was conducted at the University Hospital of Batna and a private analysis laboratory.

Results: The results of semen analysis allowed us to determine that the most common pathology in our population is OANTS with a percentage of $21.05 \%$ followed by NS and NT with 13.16\%; results of the permocytogramme show a predominance of atypical forms of the head with a frequency of $39.21 \%$. Regarding the hormone dosage, prolactin was the most disturbed hormone with an average value of $(18 \pm 6.221 \mathrm{ng} / \mathrm{ml})$, which is almost pathologic; the other hormones showed some disturbances but to a lesser degree. The results of spermiologique parameters of infertile men compared with fertile controls showed highly significant differences $(P \leq \mathrm{O} . \mathrm{OOO} 1)$ regarding motility, concentration vitality, and spermatozoa morphology. The comparison of results of hormones between the two groups revealed differences in LH and prolactin levels; no differences were found regarding FSH and testosterones. Concerning the relationship between hormones and spermiologiques parameters, positive correlations were found between (LH and sperm concentration) (testosterone and sperm concentration), and, finally, a strong correlation was found between the levels of prolactin and the anomalies of intermediate piece of spermatozoa. 
Conclusion: This study showed that hormonal disorders are not a common cause of male infertility in our study population. It further showed the relative frequencies of the various semen abnormalities during the study period. Also, we found associations between altered semen parameters and pathological levels of some hormones; however, a complementary study with a more subjects is needed.

Keywords: Male infertility, Reproductive hormones, Spermogramme, Spermocytogramme

\section{Declaration of conflicts}

This article was selected from the International Conference of Health Sciences and Medical Technologies 2016 ICHSMT'16 abstracts book.

\section{Authors' biography}

No Biography

\section{References}

No reference 